Article

\title{
Emergence of Staphylococcus lugdunensis as a Cause of Urinary Tract Infection: Results of the Routine Use of MALDI-TOF MS
}

\author{
Kelvin H. Y. Chiu ${ }^{1,+}{ }^{\dagger}$, Rex P. K. Lam ${ }^{2,+}$, Elaine Chan ${ }^{1}$, Susanna K. P. Lau ${ }^{1,3,4, *}$ and \\ Patrick C. Y. Woo 1,3,4,*iD \\ 1 Department of Microbiology, Li Ka Shing Faculty of Medicine, The University of Hong Kong, Hong Kong, \\ China; hychiu09@gmail.com (K.H.Y.C.); elaineee@gmail.com (E.C.) \\ 2 Emergency Medicine Unit, Li Ka Shing Faculty of Medicine, The University of Hong Kong, Hong Kong, \\ China; lampkrex@hku.hk \\ 3 State Key Laboratory of Emerging Infectious Diseases, The University of Hong Kong, Hong Kong, China \\ 4 Collaborative Innovation Center for Diagnosis and Treatment of Infectious Diseases, The University of Hong \\ Kong, Hong Kong, China \\ * Correspondence: skplau@hku.hk (S.K.P.L.); pcywoo@hku.hk (P.C.Y.W.); \\ Tel.: +852-2255-2584 (S.K.P.L. \& P.C.Y.W.); Fax: +852-2855-1241 (S.K.P.L. \& P.C.Y.W.) \\ + These authors contributed equally to this work.
}

Received: 20 February 2020; Accepted: 5 March 2020; Published: 9 March 2020

check for updates

\begin{abstract}
We analyzed the incidence and the clinical and laboratory characteristics of Staphylococcus lugdunensis urinary tract infections (UTIs) during a 10-year period (2009-2018) and compared them with those of Staphylococcus saprophyticus UTIs. A total of 38 and 162 episodes of S. lugdunensis and S. saprophyticus UTIs were observed. The number of S. saprophyticus UTIs was stable throughout the 10 years, whereas there was an obvious surge in the apparent number of S. lugdunensis UTIs since 2014, coinciding with the commencement of a routine use of MALDI-TOF MS. Univariate analysis showed that male sex $(p<0.001)$, advanced age $(p<0.001)$, hospital-acquired infections, $(p<0.001)$, upper UTI $(p<0.005)$, polymicrobial infections $(p<0.05)$, hypertension $(p<0.001)$, solid-organ malignancies $(p<0.001)$, renal stones $(p<0.001)$, urinary stricture $(p<0.05)$, vesicoureteral reflux $(p<0.001)$, and presence of a urinary catheter $(p<0.001)$ were significantly associated with $S$. lugdunensis UTI. Multivariable analysis revealed that $S$. lugdunensis UTI was associated with male sex $(\mathrm{OR}=6.08, p<0.05)$, solid-organ malignancies $(\mathrm{OR}=12.27, p<0.01)$, and urological system abnormalities ( $\mathrm{OR}=7.44, p<0.05)$. There were significant differences in the patient population affected and predisposing factors between S. lugdunensis and S. saprophyticus UTIs.
\end{abstract}

Keywords: Staphylococcus lugdunensis; urinary tract infection; MALDI-TOF MS

\section{Introduction}

Traditionally, staphylococci have been classified phenotypically by the presence or absence of coagulase. This method is also used to identify individual staphylococcus strains such as coagulase-positive Staphylococcus aureus or coagulase-negative staphylococci. Among the coagulase-negative staphylococci, Staphylococcus saprophyticus, identified phenotypically by its resistance to novobiocin, is the second most important cause of community-acquired urinary tract infection (UTI) in sexually active young females [1]. Apart from S. saprophyticus, other coagulase-negative staphylococci isolated from the urinary tract were often not further identified to the species level, and therefore their roles in UTI cannot be ascertained. In recent years, another coagulase-negative staphylococcus, Staphylococcus lugdunensis, has emerged as a cause of invasive bacterial infections, such as infective endocarditis and skin and soft tissue 
infections [2]. It is of note that both S. lugdunensis and S. saprophyticus reside around the genitourinary tract, with S. lugdunensis colonizing the pelvic, perineum, and groin region, and S. saprophyticus colonizing the rectum and genitourinary tract [3]. Considering their similar localization in the human body as well as their important virulence properties, we hypothesized that $S$. lugdunensis is a previously underestimated pathogen of the urinary tract. Although case reports and small case series of S. lugdunensis UTI have been described [4-6], there has been no systematic analysis of the clinical and laboratory characteristics on this clinical entity.

Matrix-assisted laser desorption ionization time-of-flight mass spectrometry (MALDI-TOF MS) has recently emerged as a revolutionary technique for the identification of bacterial pathogens, yielding rapid, accurate, and highly reproducible results at a lower price than any other methods routinely used in clinical laboratories [7]. The methodology is easy to follow, requires only a minimal amount of bacteria for the analysis, and provides results within minutes. Therefore, it has now been integrated into many clinical laboratories and is useful for the identification of different groups of medically important bacteria [8-10]. Since the start of the routine use of MALDI-TOF MS in our clinical microbiology laboratory in January 2014, we have observed an unprecedented surge of S. lugdunensis isolates identified in urine samples of our patients. In this study, we describe the apparent emergence and the clinical and laboratory characteristics of patients with UTIs caused by S. lugdunensis in a 10 -year period and compare them with those of S. saprophyticus in the same study period.

\section{Materials and Methods}

\subsection{Ethical Statement}

This study was approved by the Institutional Review Board of The University of Hong Kong/Hospital Authority (UW16-365, approved 22-07-2016).

\subsection{Patients}

All patients whose urine samples were found positive to S. lugdunensis and S. saprophyticus during a 10-year period (2009-2018) in hospitals in Hong Kong West Cluster-including Queen Mary Hospital, Tung Wah hospital, Grantham Hospital, and Fung Yiu King Hospital of Hong Kong-were included in the study. The medical records of the patients were retrieved for analysis. Those episodes of illness that met the definition of UTI given below were included for further analysis (Figure 1).

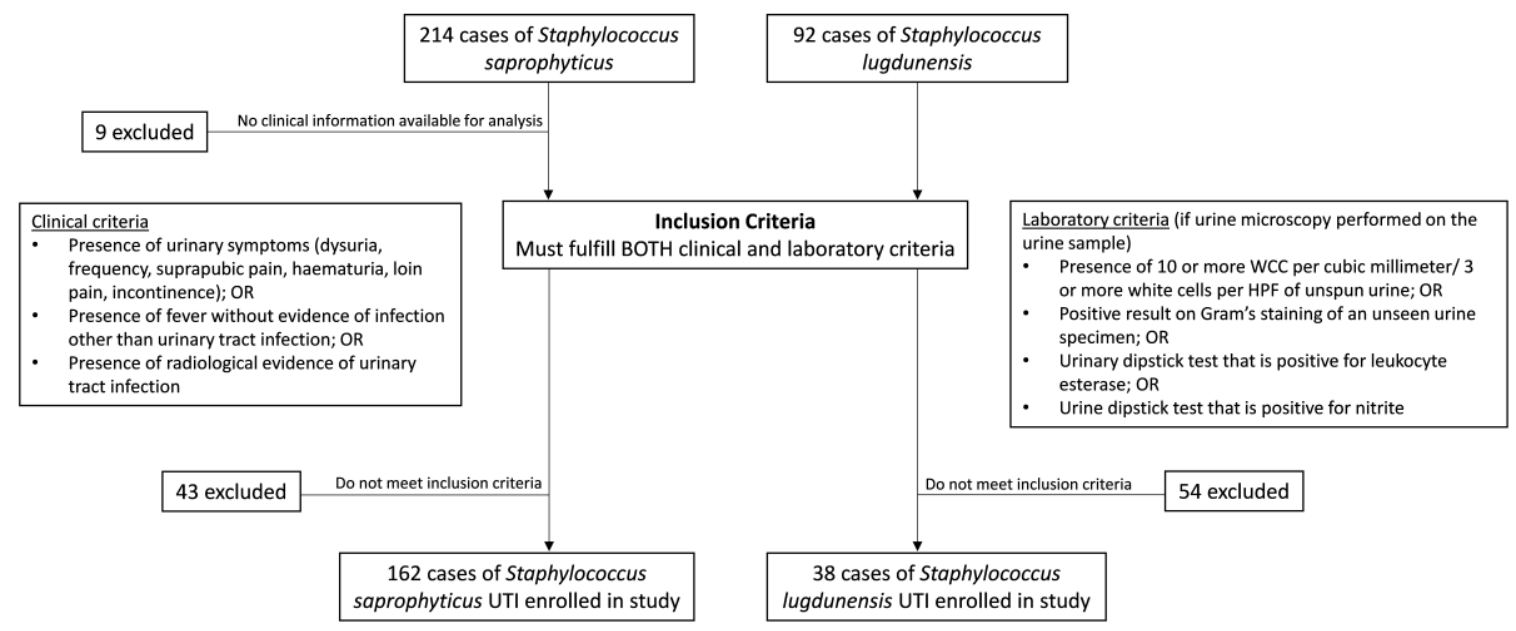

Figure 1. Enrollment flowchart of urinary tract infection (UTI) cases caused by Staphylococcus saprophyticus and Staphylococcus lugdunensis in 2009-2018. 


\subsection{Microbiological Methods}

Before January 2014, all staphylococci were first tested with Staphaurex plus latex agglutination test (Thermo Scientific). For all coagulase-negative staphylococci, the novobiocin disk diffusion test was used to identify S. saprophyticus (novobiocin-resistant). All novobiocin-sensitive coagulase-negative staphylococci were discarded, unless the microbiologist had requested their full identification. After January 2014, all staphylococci were preliminarily identified by MALDI-TOF MS. When MALDI-TOF MS results showed the presence of S. lugdunensis, bacterial identity was further confirmed by positive Staphaurex plus latex agglutination test and negative tube coagulase test. When the MALDI-TOF MS results showed the presence of $S$. saprophyticus, bacterial identity was further confirmed by negative Staphaurex plus latex agglutination test and resistance to novobiocin.

\subsection{Definitions}

UTI was defined based on the fulfillment of both clinical and laboratory criteria. Clinical identification included the fulfillment of at least one of the following criteria: presence of urinary symptoms (dysuria, urinary frequency, suprapubic pain, gross hematuria, loin pain, urinary incontinence); fever without other culture result suggestive of infection other than UTI; and radiological evidence of UTI. Laboratory criteria included the isolation at least $10^{4}$ colony-forming unit (cfu) per $\mathrm{mL}$ of urine of the target microorganism and, if urine microscopy or biochemical tests were performed, the presence of pyuria, determined on the basis of either one of the following: 10 or more white blood cells per $\mathrm{mL}, 3$ or more white blood cells per high-power field of unspun urine, presence of bacteria identified by direct microscopy, positivity for leukocyte esterase or nitrite in the urine dipstick test.

\subsection{Statistical Analysis}

Categorical variables were analyzed by Fisher's exact test or Chi-square test, and continuous variables were analyzed by Mann-Whitney U test using SPSS version 24.0 (IBM Corp., Armonk, NY, USA). A $p$ value $<0.05$ was considered statistically significant. Variables that were considered as statistically significant in univariate analysis were subjected to multivariable analysis by binomial logistic regression.

\section{Results}

\subsection{Emergence of UTI Caused by S. lugdunensis}

From January 2009 to December 2018, a total of 306 non-duplicated S. lugdunensis and S. saprophyticus isolates were recovered from the urine samples of 289 patients. Two hundred (from 190 patients) of the 306 episodes fulfilled the diagnostic criteria of UTI and were subjected to further analysis. Among these 200 episodes, 38 were S. lugdunensis and 162 were S. saprophyticus infections. The number of $S$. saprophyticus UTI episodes was stable throughout the 10 years, whereas there was an obvious surge in the apparent number of S. lugdunensis UTI episodes since 2014, coinciding with the commencement of the use of MALDI-TOF MS in our clinical microbiology laboratory (Figure 2). In fact, there was only one S. lugdunensis UTI episode from 2009 to 2013, whereas there were 38 S. lugdunensis UTI episodes from 2014 to 2018. 


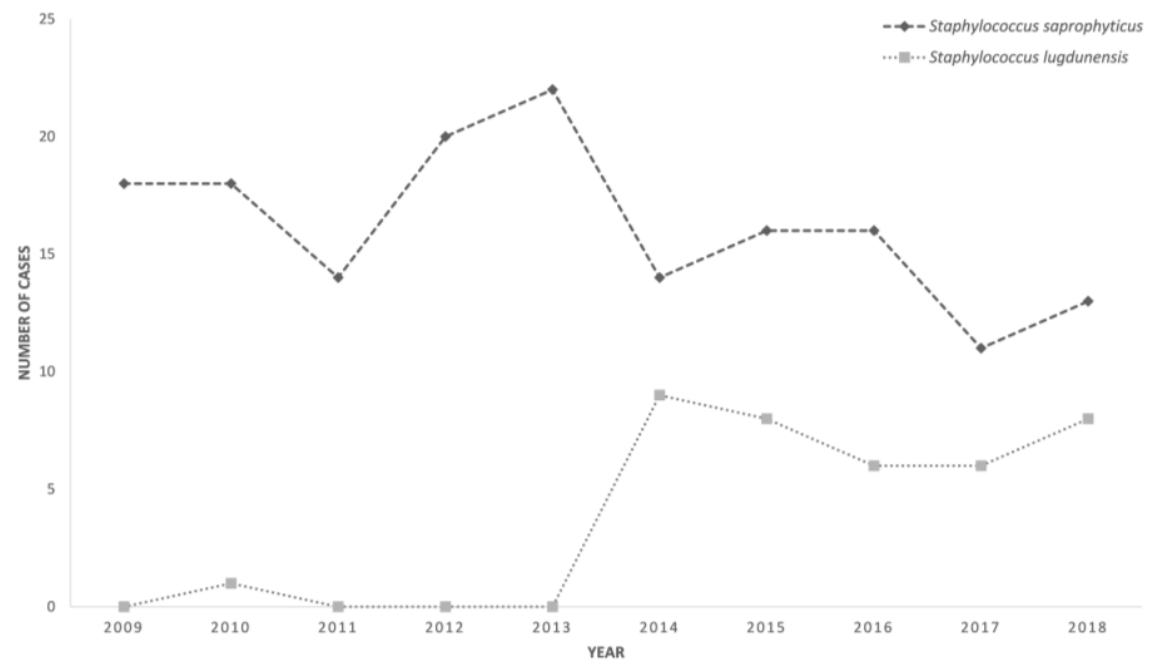

Figure 2. Cases of S. saprophyticus- and S. lugdunensis-associated urinary tract infection in 2009-2018.

\subsection{Characteristics of Patients with S. lugdunensis UTI}

Patients with S. lugdunensis UTI were predominantly male $(71.1 \%)$, whereas only $3.1 \%$ of those with $S$. saprophyticus UTI were male $(p<0.001)$. The median age of patients with S. lugdunensis UTI was 59 (range 27-90), significantly higher than that of patients with S. saprophyticus UTI (median 29, range 4-90) $(p<0.001)$ (Table 1). There was no obvious seasonal variation for S. lugdunensis UTI (Figure 3). In addition, $15.8 \%$ of S. lugdunensis UTIs were hospital-acquired infections, whereas only $1.9 \%$ of $S$. saprophyticus UTIs were hospital-acquired $(p<0.001) ; 15.8 \%$ of $S$. lugdunensis UTIs were upper urinary tract infections, whereas only $2.5 \%$ of $S$. saprophyticus UTIs were upper urinary tract infections $(p<0.005)$. None of the patients with S. lugdunensis UTI developed S. lugdunensis bacteremia, and none of them died during the episode of UTI.

Table 1. Clinical characteristics of S. lugdunensis and S. saprophyticus urinary tract infection.

\begin{tabular}{cccc}
\hline Characteristics & $\begin{array}{c}\text { S. lugdunensis UTI } \\
(\boldsymbol{n}=\mathbf{3 8})\end{array}$ & $\begin{array}{c}\text { S. saprophyticus } \\
\text { UTI }(\boldsymbol{n = 1 6 2 )}\end{array}$ & $p$ Value \\
\hline Sex (male) & $27(71.1)$ & $5(3.1)$ & $<0.001$ \\
Age (median, range) & $59.0(27-90)$ & $29.0(4-90)$ & $<0.001^{1}$ \\
Hospital-acquired & $6(15.8)$ & $3(1.9)$ & 0.002 \\
Upper UTI & $6(15.8)$ & $4(2.5)$ & 0.004 \\
Polymicrobial infection & $10(26.3)$ & $18(11.1)$ & 0.015 \\
Local predisposing factors and urological system abnormalities & \\
Renal stone & $9(23.7)$ & $5(3.1)$ & $<0.001$ \\
Urinary stricture & $3(7.9)$ & $1(0.6)$ & 0.022 \\
Vesicoureteral reflux & $3(7.9)$ & $0(0.0)$ & 0.006 \\
Renal transplantation & $1(2.6)$ & $0(0.0)$ & 0.190 \\
Urinary catheter & $13(34.2)$ & $3(1.9)$ & $<0.001$ \\
Other underlying illness & & & \\
Hypertension & $15(39.5)$ & $8(4.9)$ & $<0.001$ \\
Solid-organ malignancy & $17(44.7)$ & $2(1.2)$ & 0.190 \\
Hematological malignancy & $1(2.6)$ & $0(0.0)$ & 0.376 \\
Diabetes mellitus & $3(7.9)$ & $6(3.7)$ & 0.405 \\
Immunodeficiency & $3(7.9)$ & $7(4.3)$ & 0.164 \\
Cerebrovascular accident & $2(5.3)$ & $2(1.2)$ & 0.093 \\
End-stage renal failure & $2(5.3)$ & $1(0.6)$ & \\
\hline
\end{tabular}

Data are presented as No. (\%) unless otherwise indicated. All statistical analyses were carried out by the Fisher's exact test unless otherwise indicated. ${ }^{1}$ Calculated by Mann-Whitney U test. 


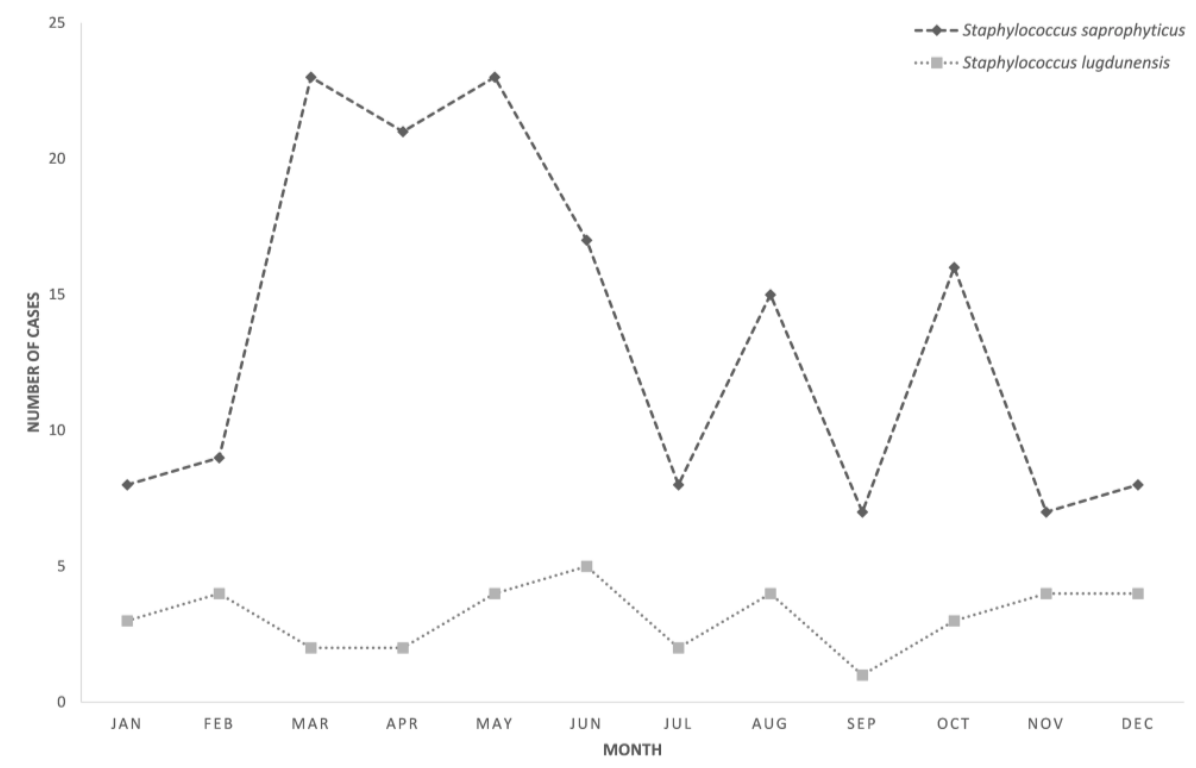

Figure 3. Cases of S. saprophyticus- and S. lugdunensis-associated urinary tract infection recorded by month of specimen collection (2009 - 2018).

Patients with $S$. lugdunensis UTI were significantly more likely to have co-existing bacteria in the urine than those with S. saprophyticus UTI $(26.3 \%$ and $11.1 \%$, respectively, $p<0.05)$. The three commonest co-existing organisms isolated from the urine of S. lugdunensis UTI patients were Enterococcus species $(40.0 \%)$, coagulase-negative staphylococci other than S. saprophyticus $(30.0 \%)$, and Escherichia coli (20.0\%). The three commonest co-existing organisms isolated from the urine of S. saprophyticus UTI patients were E. coli (44.4\%), Corynebacterium species (11.1\%), and Citrobacter species (11.1\%).

As for the association with general underlying diseases, the incidences of hypertension and solid-organ malignancies in patients with S. lugdunensis UTI (39.5\% and $44.7 \%$, respectively) were significantly higher than those in patients with S. saprophyticus UTI (4.9\% and $1.2 \%$, respectively) $(p<0.001$ and $p<0.001$, respectively). As for local predisposing factors and urological system abnormalities, the incidences of renal stones, urinary stricture, vesicoureteral reflux, and presence of a urinary catheter in patients with S. lugdunensis UTI $(23.7 \%, 7.9 \%, 7.9 \%$, and $34.2 \%$, respectively) were significantly higher than those in patients with S. saprophyticus UTI $(3.1 \%, 0.6 \%, 0 \%$ and $1.9 \%$, respectively) ( $p<0.001, p<0.05, p<0.001$ and $p<0.001$, respectively).

Multivariable analysis using binomial logistic regression revealed that $S$. lugdunensis UTI was associated with male sex (odds ratio $[\mathrm{OR}]=6.08, p<0.05)$, solid-organ malignancies $(\mathrm{OR}=12.27$, $p<0.01)$, and urological system abnormalities $(\mathrm{OR}=7.44, p<0.05)$ (Table 2$)$.

Table 2. Results of a multivariable analysis of the clinical characteristics associated with S. lugdunensis and $S$. saprophyticus urinary tract infection.

\begin{tabular}{lccc}
\hline & \multicolumn{3}{c}{ Multivariable Analysis } \\
\cline { 2 - 4 } Characteristics & OR & $\mathbf{9 5 \% ~ C I ~}$ & $p$ Value \\
\hline Sex (male) & 6.08 & $1.26-29.43$ & 0.025 \\
Age (median, range) & 1.03 & $0.99-1.07$ & 0.115 \\
Hospital acquired & 3.72 & $0.52-26.82$ & 0.193 \\
Upper UTI & 6.69 & $0.46-98.18$ & 0.165 \\
Local predisposing factors and urological system abnormalities ${ }^{1}$ & 7.44 & $1.52-36.37$ & 0.013 \\
Hypertension & 3.62 & $0.60-21.70$ & 0.159 \\
Solid-organ malignancy & 12.27 & $1.800-83.69$ & 0.010 \\
\hline All statistical analyses were carried out by the Fisher's exact test unless otherwise indicated. CI, confidence interval; \\
OR, odds ratio. ${ }^{1}$ Includes incidence of renal stones, urinary stricture, vesicoureteral reflux, renal transplantation, \\
and presence of a urinary catheter.
\end{tabular}




\subsection{Recurrent S. lugdunensis UTI}

Two of the 38 patients had recurrence of S. lugdunensis UTI. The first patient was a 69-year-old Chinese man with a carcinoma of the bladder diagnosed six years before, who was treated with transurethral resection of the bladder tumor and intravesical bacillus Calmette-Guerin (BCG) therapy. He presented with dysuria that had been ongoing for two days, with turbid urine. S. lugdunensis (susceptible to ciprofloxacin, erythromycin, methicillin, nitrofurantoin, cotrimoxazole, gentamicin, and vancomycin) was recovered from his urine. Subsequent investigation revealed right vesico-ureteric junction narrowing, with vesicoureteral reflux. He was given oral amoxicillin-clavulanate for one week. However, the symptoms recurred 11 days after stopping the antibiotic. At that time, S. lugdunensis with a different antibiotic susceptibility profile (susceptible to ciprofloxacin, erythromycin, methicillin, nitrofurantoin, cotrimoxazole, and vancomycin but resistant to gentamicin) was recovered from his urine. He was given oral amoxicillin-clavulanate again for one week, and there were no more recurrences of S. lugdunensis UTI.

The second patient was a 53-year-old Chinese man with underlying left renal stone and right ureteric stone, with right-sided hydronephrosis. Extracorporeal shock wave lithotripsy and subsequent ureterorenoscopic lithotripsy was performed one year before presentation. He initially presented with bilateral loin discomfort. Urine microscopy showed abundant white blood cells, and urine culture recovered a pure growth of $>10^{5} \mathrm{cfu} / \mathrm{mL}$ S. lugdunensis (susceptible to nitrofurantoin and cotrimoxazole but resistant to methicillin and gentamicin). Antibiotics treatment was not administered, but the patient's symptoms gradually subsided. Three months later, extracorporeal shock wave lithotripsy was repeated, and the patient developed fever and bilateral loin discomfort four days later. Computed tomography showed a renal stone at the left pelvo-ureteric junction, with hydronephrosis. A left JJ stent was inserted. Microscopic examination of the ureteric urine showed abundant leukocytes, and urine culture recovered S. lugdunensis with a different antibiotic susceptibility profile (susceptible to methicillin, nitrofurantoin, and cotrimoxazole but resistant to gentamicin). He was given intravenous and then oral cefuroxime for one week, which stopped the recurrence of S. lugdunensis UTI.

\section{Discussion}

This is the first study analyzing the clinical and laboratory characteristics of S. lugdunensis UTI systematically. Upon analysis of all patients with S. lugdunensis UTI in the last 10 years, it was observed that there was an apparent emergence of UTI caused by S. lugdunensis since 2014 (Figure 2). From 2009 to 2014, when S. lugdunensis was identified by positive Staphaurex plus latex agglutination test and negative tube coagulase test, only one case of $S$. lugdunensis UTI was recorded. This was because S. lugdunensis was not recognized as an important urinary tract pathogen, and coagulase-negative staphylococci that were not $S$. saprophyticus were not further identified to the species level in the past. The marked increase in the detection of S. lugdunensis isolates in urine samples since 2014 coincided with the commencement of using MALDI-TOF MS for rapid bacterial identification in our clinical microbiology laboratory. The use of MALDI-TOF MS has facilitated the identification of a number of bacteria in our laboratory, such as Burkholderia pseudomallei, Laribacter hongkongensis, and Tsukamurella species [8-10]. This method not only increases the number of cases diagnosed but also shortens the time to diagnosis. In the past, when conventional methods were used for bacteria identification, it took at least $24 \mathrm{~h}$ to confirm the identities of most bacteria, whereas MALDI-TOF MS only requires a few minutes. This improvement in the laboratory diagnosis of $S$. lugdunensis UTI gave us a good opportunity to analyze the epidemiology, predisposing factors, clinical spectrum, and infection outcome of this distinct disease entity. In contrast to S. lugdunensis, there have been no changes in the incidence of $S$. saprophyticus UTI in the last 10 years, though MALDI-TOF MS can shorten the time of identification of this bacterium as well.

There were significant differences in the patient population affected and the predisposing factors between S. lugdunensis and S. saprophyticus UTIs. Similar to what was described in the literature [11], the present cohort of patients with S. saprophyticus UTI were mainly young and healthy females 
with lower UTI. On the other hand, patients with S. lugdunensis UTI were predominantly males and much older (Table 1). A significant proportion of S. lugdunensis UTI patients had underlying diseases, most importantly solid-organ malignancies and urological abnormalities (Table 1). Solid-organ malignancies associated with $S$. lugdunensis UTI, 53.8\% involved the genitourinary system [bladder $(n=4)$, prostate $(n=4)$, kidney $(n=1)$, ovary $(n=1)$, vagina $(n=1)]$, whereas the most common urological abnormalities included the presence of renal stones, urinary stricture, and vesicoureteral reflux. It is of note that the two patients in this cohort with recurrent S. lugdunensis UTI had underlying urological abnormalities: carcinoma of the bladder and vesicoureteral reflux in the first patient, and presence of renal stones in the second patient. These urological abnormalities enhance the colonization of pathogenic bacteria and increase the difficulty in clearing them. After all, although patients with $S$. lugdunensis were older with underlying diseases, none of them developed bacteremia or died during the corresponding episodes of UTI, indicating tha S. lugdunensis UTI is still a relatively benign and readily treatable infection.

The pathogenesis of S. lugdunensis UTI remains to be determined. S. saprophyticus is primarily a pathogen of the urinary tract, and the factors involved in the mediation of UTI caused by S. saprophyticus are relatively well studied. S. saprophyticus possesses various types of adhesins such as hemagglutinins, autolysin Ass, and surface-associated lipases such as Ssp, which facilitate effective bacterial adherence to fibronectin and fibrinogen and, hence, colonization of the uroepithelium [12-14]. It can also form a bacterial biofilm, which confers an anti-phagocytic and antimicrobial-resistance phenotype and increases the virulence of the bacteria [15]. Furthermore, S. saprophyticus is urease-positive, which is important for bacterial colonization and invasion. Essentially, urease catalyzes the hydrolysis of urea to carbon dioxide and ammonia, which results in an increase in urine $\mathrm{pH}$ and the production of calcium and magnesium phosphate crystals in the urine. This accumulation of ammonia is toxic to uroepithelial cells and causes direct tissue damage [16]. As for S. lugdunensis, its virulence properties were often studied in the context of other infections that it can cause, such as infective endocarditis and catheter-related bacteremia [17]. S. lugdunensis does not produce urease but does encode a $\delta$-like hemolysin that shares phenotypic properties with the S. aureus delta-toxin [18]. The S. aureus-produced delta-toxin can attach non-specifically to the cytoplasmic membrane of a cell and form short-lived membrane-damaging pores which cause cell lysis and subsequent cell death. In addition, S. lugdunensis encodes the staphylococcal agr locus, a quorum-sensing locus that regulates a number of virulence genes such as enterotoxins and hemolysins [19], and a S. lugdunensis synergistic hemolysin (slush) locus, which encodes three highly similar 43-amino-acid hemolytic peptides with delta-toxin-like activity [20]. These hemolytic proteins may potentially contribute to the invasion of the bacteria and cause tissue damage as observed for urease activity. Moreover, S. lugdunensis encodes host surface adherence proteins such as the multifunctional autolysin/adhesin AtlL, which binds to the extracellular matrix, to plasma proteins such as fibronectin and fibrinogen, and to human endothelial cells [21]. Mutation of AtlL also result in a significantly reduced ability of the bacteria to form biofilm. We speculate that these virulence factors may mediate $S$. lugdunensis UTI in a similar way as that observed for $S$. saprophyticus, via adherence and colonization of the uroepithelium and biofilm formation, particularly in the presence of urinary catheters on which biofilm can easily form. Underlying urological abnormalities that lead to stagnation of urine are important for S. lugdunensis multiplication. Further studies are required to delineate which of these virulence factors are particularly important for the pathogenesis of S. lugdunensis UTI.

\section{Conclusions}

An apparent surge in the number of S. lugdunensis UTI was observed since 2014, which coincided with the start of the routine use of MALDI-TOF MS in our clinical microbiology laboratory. In comparison with UTI caused by $S$. saprophyticus, significant differences in the patient population affected and predisposing factors were found for S. lugdunensis UTI. 
Author Contributions: Conceptualization, P.C.Y.W.; methodology, K.H.Y.C., S.K.P.L., and P.C.Y.W.; formal analysis, K.H.Y.C. and R.P.K.L.; writing — original draft preparation, K.H.Y.C., E.C., and P.C.Y.W.; writing-review and editing, K.H.Y.C., R.P.K.L., E.C., S.K.P.L., and P.C.Y.W.; supervision, S.K.P.L. and P.C.Y.W. All authors have read and agreed to the published version of the manuscript.

Funding: This work was funded by the University Development Fund, The University of Hong Kong, Hong Kong.

Conflicts of Interest: Patrick C. Y. Woo has provided scientific advisory/laboratory services for Gilead Sciences, Inc., International Health Management Associates, Inc./Pfizer, Inc., and Merck \& Co, Inc. All other authors have no potential conflicts.

\section{References}

1. Hovelius, B.; Mårdh, P.A. Staphylococcus saprophyticus as a common cause of urinary tract infections. Rev. Infect. Dis. 1984, 6, 328-337. [CrossRef] [PubMed]

2. Lin, J.F.; Cheng, C.W.; Kuo, A.J.; Liu, T.P.; Yang, C.C.; Huang, C.T.; Lee, M.H.; Lu, J.J. Clinical experience and microbiologic characteristics of invasive Staphylococcus lugdunensis infection in a tertiary center in northern Taiwan. J. Microbiol. Immunol. Infect. 2015, 48, 406-412. [CrossRef] [PubMed]

3. Van der Mee-Marquet, N.; Achard, A.; Mereghetti, L.; Danton, A.; Minier, M.; Quentin, R. Staphylococcus lugdunensis infections: High frequency of inguinal area carriage. J. Clin. Microbiol. 2003, 41, 1404-1409. [CrossRef] [PubMed]

4. Casanova-Roman, M.; Sanchez-Porto, A.; Casanova-Bellido, M. Urinary tract infection due to Staphylococcus lugdunensis in a healthy child. Scand. J. Infect. Dis. 2004, 36, 149-150. [CrossRef] [PubMed]

5. Hayakawa, I.; Hataya, H.; Yamanouchi, H.; Sakakibara, H.; Terakawa, T. Neonatal Staphylococcus lugdunensis urinary tract infection. Pediatr. Int. 2015, 57, 783-785. [CrossRef] [PubMed]

6. Haile, D.T.; Hughes, J.; Vetter, E.; Kohner, P.; Snyder, R.; Patel, R.; Cockerill, F.R., 3rd. Frequency of isolation of Staphylococcus lugdunensis in consecutive urine cultures and relationship to urinary tract infection. J. Clin. Microbiol. 2002, 40, 654-656. [CrossRef]

7. Schubert, S.; Kostrzewa, M. MALDI-TOF MS in the microbiology laboratory: Current trends. Curr. Issues Mol. Biol. 2017, 23, 17-20. [CrossRef]

8. Lau, S.K.; Tang, B.S.; Curreem, S.O.; Chan, T.M.; Martelli, P.; Tse, C.W.; Wu, A.K.; Yuen, K.Y.; Woo, P.C. Matrix-assisted laser desorption ionization-time of flight mass spectrometry for rapid identification of Burkholderia pseudomallei: Importance of expanding databases with pathogens endemic to different localities. J. Clin. Microbiol. 2012, 50, 3142-3143. [CrossRef]

9. Tang, B.S.; Lau, S.K.; Teng, J.L.; Chan, T.M.; Chan, W.S.; Wong, T.Y.; Tong, Y.T.; Fan, R.Y.; Yuen, K.Y.; Woo, P.C. Matrix-assisted laser desorption ionisation-time of flight mass spectrometry for rapid identification of Laribacter hongkongensis. J. Clin. Pathol. 2013, 66, 1081-1083. [CrossRef]

10. Teng, J.L.L.; Tang, Y.; Wong, S.S.Y.; Fong, J.Y.H.; Zhao, Z.; Wong, C.P.; Chen, J.H.K.; Ngan, A.H.Y.; Wu, A.K.L.; Fung, K.S.C.; et al. MALDI-TOF MS for identification of Tsukamurella species: Tsukamurella tyrosinosolvens as the predominant species associated with ocular infections. Emerg. Microbes Infect. 2018, 7, 80. [CrossRef]

11. Lo, D.S.; Shieh, H.H.; Barreira, E.R.; Ragazzi, S.L.; Gilio, A.E. High frequency of Staphylococcus saprophyticus urinary tract infections among female adolescents. Pediatr. Infect. Dis. J. 2015, 34, 1023-1025. [CrossRef] [PubMed]

12. Raz, R.; Colodner, R.; Kunin, C.M. Who are you-Staphylococcus saprophyticus? Clin. Infect. Dis. 2005, 40, 896-898. [CrossRef] [PubMed]

13. Meyer, H.G.; Wengler-Becker, U.; Gatermann, S.G. The hemagglutinin of Staphylococcus saprophyticus is a major adhesin for uroepithelial cells. Infect. Immun. 1996, 64, 3893-3896. [CrossRef] [PubMed]

14. Szabados, F.; Mohner, A.; Kleine, B.; Gatermann, S.G. Staphylococcus saprophyticus surface-associated protein (Ssp) is associated with lifespan reduction in Caenorhabditis elegans. Virulence 2013, 4, 604-611. [CrossRef]

15. Martins, K.B.; Ferreira, A.M.; Pereira, V.C.; Pinheiro, L.; de Oliveira, A.; da Cunha, M.L.R.S. In vitro effects of antimicrobial agents on planktonic and biofilm forms of Staphylococcus saprophyticus isolated from patients with urinary tract infections. Front. Microbiol. 2019, 10, 40. [CrossRef]

16. Flores-Mireles, A.L.; Walker, J.N.; Caparon, M.; Hultgren, S.J. Urinary tract infections: Epidemiology, mechanisms of infection and treatment options. Nat. Rev. Microbiol. 2015, 13, 269-284. [CrossRef]

17. Frank, K.L.; Del Pozo, J.L.; Patel, R. From clinical microbiology to infection pathogenesis: How daring to be different works for Staphylococcus lugdunensis. Clin. Microbiol. Rev. 2008, 21, 111-133. [CrossRef] 
18. Vandenesch, F.; Storrs, M.J.; Poitevin-Later, F.; Etienne, J.; Courvalin, P.; Fleurette, J. Delta-like haemolysin produced by Staphylococcus lugdunensis. FEMS Microbiol. Lett. 1991, 62, 65-68.

19. Dufour, P.; Jarraud, S.; Vandenesch, F.; Greenland, T.; Novick, R.P.; Bes, M.; Etienne, J.; Lina, G. High genetic variability of the agr locus in Staphylococcus species. J. Bacteriol. 2002, 184, 1180-1186. [CrossRef]

20. Donvito, B.; Etienne, J.; Denoroy, L.; Greenland, T.; Benito, Y.; Vandenesch, F. Synergistic hemolytic activity of Staphylococcus lugdunensis is mediated by three peptides encoded by a non-agr genetic locus. Infect. Immun. 1997, 65, 95-100. [CrossRef]

21. Hussain, M.; Steinbacher, T.; Peters, G.; Heilmann, C.; Becker, K. The adhesive properties of the Staphylococcus lugdunensis multifunctional autolysin AtlL and its role in biofilm formation and internalization. Int. J. Med. Microbiol. 2015, 305, 129-139. [CrossRef] [PubMed]

(C) 2020 by the authors. Licensee MDPI, Basel, Switzerland. This article is an open access article distributed under the terms and conditions of the Creative Commons Attribution (CC BY) license (http://creativecommons.org/licenses/by/4.0/). 